\title{
Cost of a population-based programme of chest $x$-ray screening for lung cancer
}

\author{
L. Dominioni1 ${ }^{1}$ N. Rotolo1, A. Poli2, M. Castiglioni1 ${ }^{1}$, M. Mangini3 3 , M. Spagnoletti ${ }^{1}$, \\ M. Paolucci4, A. Paddeu5, W. Mantovani6, A. Zanini7, A. Imperatori ${ }^{1}$
}

\begin{abstract}
Cost of a population-based programme of chest $x$-ray screening for lung cancer. L. Dominioni, $N$. Rotolo, A. Poli, M. Castiglioni, M. Mangini, M. Spagnoletti, M. Paolucci, A. Paddeu, W. Mantovani, A. Zanini, A. Imperatori.

Background. After the implementation of a population-based programme of chest $\mathrm{x}$-ray (CXR) screening on smokers in Varese, Italy, lung cancer (LC) mortality was significantly reduced. Analysis of the incremental costs due to this type of screening programme is needed to evaluate its economic impact on the healthcare system.

Methods. In July 1997 a population-based cohort, consisting of all high-risk smokers $(n=5,815)$ identified among 60,000 adult residents from the Varese province, was invited to a LC screening programme (an annual CXR for five years) in a general practice setting, and was observed through 2006. Invitees received National Health Service (NHS) usual care, with the addition of CXRs in screening participants. At the end of observation, among the 245 LCs diagnosed in the entire screening-invited co-
\end{abstract}

hort the observed LC deaths were 38 fewer than expected. To estimate the incremental direct cost due to screening in the invited cohort for the period July 1997-2006, we compared the direct cost of screening administration, CXR screens and LC management in the invited cohort and in the uninvited and unscreened controls in NHS usual care setting.

Results. Over the 9.5 years, the total incremental direct healthcare costs (including screening organization/administration, CXR screens, additional procedures prompted by false-positive tests, overdiagnosed LCs) were estimated to range from $€ 607,440$ to $€ 618,370$ (in euros as of 2012), equating to between $€ 15,985-€ 16,273$ per patient out of the $38 \mathrm{LC}$ deaths averted.

Conclusions. In a general practice setting, the incremental cost for a CXR screening programme targeted at all high-risk smokers in a population of 60,000 adults was estimated to be about $€ 65,000$ per annum, approx. $€ 16,000$ for each LC death averted.

Monaldi Arch Chest Dis 2013; 79: 2, 67-72.

Keywords: Lung cancer screening, Economic evaluation, Chest radiography, Chest $x$-ray, Population-based, Cost-effectiveness.

1 Center for Thoracic Surgery, University of Insubria, Ospedale di Circolo di Varese;

2 Department of Public and Community Medicine, University of Verona;

3 Department of Radiology, University of Insubria, Ospedale di Circolo di Varese;

4 Department of Radiology, Ospedale S. Antonio Abate, Gallarate;

5 Respiratory Care Unit, Ospedale S. Anna, San Fermo della Battaglia, Como;

6 Department of Prevention, Public Health Trust, Trento;

7 Division of Pneumology, Fondazione F. Maugeri, IRCCS Rehabilitation Institute of Tradate, Italy.

Correspondence: Lorenzo Dominioni, Center for Thoracic Surgery, University of Insubria, Ospedale di Circolo, Via Guicciardini 9 , 21100Varese, Italy; e-mail:lorenzo.dominioni@uninsubria.it

\section{Introduction}

With the aim to contribute to the assessment of the effectiveness of chest X-ray (CXR) screening for lung cancer (LC), during the 1997-2006 period we carried out an observational study of CXR screening on a clearly defined populationbased cohort. This project was coordinated by the Center for Thoracic Surgery of the University of Insubria, Varese, Italy and was funded by NonProfit Organizations. The study was approved by the Varese Hospital and Health District Ethics Committee and was registered under the registration code: ISRCTN90639073. The study cohort, consisting of 5,815 smokers all from the Varese Province, was invited to undergo a free-of-charge programme of annual CXR screenings over a pe- riod of 5 years, and was recruited by 50 general practitioners and physicians from a population of about 60,000 adult residents from the greater Varese provincial area. The entire invited cohort received National Health Service (NHS) usual care, with the addition of CXRs on the screening participants (21\% of the invitees), according to the previously described protocol [1]. At the end of the screening programme, a total of $245 \mathrm{LCs}$ were found out of the entire screening cohort (67 in participants, 178 in non-participants), of which 34 were screen-detected [1]. The LC standardized mortality ratio (SMR), for the entire screeninginvited cohort, was of 0.82 , indicating in the cohort a LC mortality reduction of $18 \%$, and 38 fewer LC deaths over the study period [2]. This is a relatively small benefit, however it could save 
many lives, especially given the high incidence of LC worldwide and the potentially wide applicability of CXR screening. In order to explore the NHS budget impact of a population-based LC screening intervention, it is necessary to evaluate the direct costs involved, the benefits as well as the harms. Here we describe the economic evaluation of the annual CXR screening programme we carried out in the Varese province in a general practice setting.

\section{Methods}

We aimed to evaluate the incremental costs attributable to the programme of CXR screening for LC which we performed from July 1997 through December 2006. To this effect, over the 9.5-year duration of the screening programme we analyzed the total direct costs of CXR screening and LC management in the screening-invited cohort, and compared it with the total direct cost of LC man-

Table 1. - Cost of diagnosis and staging of lung cancer (LC)

\begin{tabular}{lc}
\hline Components of cost & Cost in Euros* \\
\hline Screening & \\
CXR exam, dual projection & 15.49 \\
SUB-TOTAL & $\mathbf{1 5 . 4 9}$ \\
Cyto-/histologic confirmation of LC & \\
Bronchoscopy with biopsy & 180.76 \\
Cytology of sputum/pleural fluid & 26.86 \\
CT-guided fine needle aspirate & \\
and cytology & 157.52 \\
Hospital stay & 624.00 \\
SUB-TOTAL & 989.14 \\
Staging of LC patient stage I-IV & \\
Blood cells count, chemistry, & \\
tumor markers, arterial gases & 117.25 \\
EKG & 11.36 \\
CXR exam, dual projection & 15.49 \\
CT total body with contrast & 482.37 \\
Bone scintigraphy & 80.57 \\
Spirometry & 23.24 \\
Hospital stay & $4,160.00$ \\
SUB-TOTAL & $4,890.28$ \\
Staging of patient unfit & \\
for active treatment & \\
Blood cells count, chemistry, & \\
tumor markers, arterial gases & \\
EKG & \\
CXR exam, dual projection & 117.25 \\
CT total body with contrast & 11.36 \\
Hospital stay & 15.49 \\
SUB-TOTAL & $2,496.00$ \\
\hline
\end{tabular}

CT: computed tomography. CXR: chest X-ray. EKG: electrocardiogram. LC: lung cancer.

* Cost in Euros 2002. agement in a control group of patients observed in the general population of the same area in the absence of screening.

Throughout this paper the values of healthcare services, procedures and interventions were reported in Euros as of the year 2002, the central year of our screening study (tables 1-3). These values were estimated based on the 2002 Tariff of Lombardy Regional Bulletin and on data provided by the Oncology, Radiotherapy and Palliative Care Units at the Varese Ospedale di Circolo. For a more direct comparison with the most recently published literature, the incremental costs incurred with screening were expressed in terms of Euros as of 2012.

\section{Cost of the screening programme}

Computation of direct costs attributable to the screening programme included: a) the cost of organization and administration; b) the cost of CXR

Table 2. - Cost of treatment of lung cancer (LC)

\begin{tabular}{|c|c|}
\hline Components of cost & Cost in Euros* \\
\hline \multicolumn{2}{|l|}{ Chest surgery } \\
\hline \multicolumn{2}{|l|}{$\begin{array}{l}\text { Operating room occupancy } \\
\text { (average } 3 \text { hours) }\end{array}$} \\
\hline Operating room materials & $388.00-1,165.00$ \\
\hline $\begin{array}{l}\text { Hospital stay in surgical unit } \\
\text { (average } 12 \text { days) }\end{array}$ & $5,827.00$ \\
\hline SUB-TOTAL & $7,380.00-9,323.00$ \\
\hline \multicolumn{2}{|l|}{ Chemotherapy for LC stage III } \\
\hline 6 cycles (Cisplatin + Gemcitabine) & $4,177.00$ \\
\hline SUB-TOTAL & $4,177.00$ \\
\hline \multicolumn{2}{|l|}{ Chemotherapy for LC stage IV } \\
\hline 3 cycles (Cisplatin + Gemcitabine) & $2,089.00$ \\
\hline SUB-TOTAL & $2,089.00$ \\
\hline \multicolumn{2}{|l|}{ Palliative radiotherapy } \\
\hline Radiotherapy & $2,405.00-3,385.00$ \\
\hline SUB-TOTAL & $2,405.00-3,385.00$ \\
\hline \multicolumn{2}{|l|}{ Radical radiotherapy } \\
\hline Radiotherapy (50-60 Gy) & $2,405.00-6,974.00$ \\
\hline SUB-TOTAL & $2,405.00-6,974.00$ \\
\hline \multicolumn{2}{|l|}{ Chemo-Radiotherapy combined } \\
\hline Chemotherapy & $2,089.00-4,177.00$ \\
\hline Radiotherapy & $2,405.00-6,974.00$ \\
\hline SUB-TOTAL & $4,494.00-11,151.00$ \\
\hline \multicolumn{2}{|l|}{ Supportive/palliative therapy } \\
\hline Oxygen & 397.44 \\
\hline Drugs (analgesics, antibiotics, steroid & ds) $\quad 699,00$ \\
\hline Hospital stay & $1,040.00$ \\
\hline SUB-TOTAL & $2,136.00$ \\
\hline \multicolumn{2}{|l|}{ Terminal phase } \\
\hline Cumulative cost of 1 month & $1,399.00$ \\
\hline SUB-TOTAL & $1,399.00$ \\
\hline
\end{tabular}

LC: lung cancer.

* Cost in Euros 2002. 
Table 3. Cost of follow-up after treatment of lung cancer (LC)

\begin{tabular}{lc}
\hline Components of cost & Cost in Euros* \\
\hline First year of follow-up & \\
3 Visits & 58.00 \\
2 CXR exam, dual projection & 30.98 \\
2 Blood cells count, chemistry, & \\
tumor markers, arterial gases & 234.50 \\
1 Chest CT with contrast & 161.13 \\
SUB-TOTAL & 484.61 \\
Second year of follow-up & \\
2 Visits & 38.00 \\
1 CXR exam, dual projection & 15.49 \\
2 Blood cells count, chemistry, & \\
tumor markers, arterial gases & 234.50 \\
1 Chest CT with contrast & 161.13 \\
SUB-TOTAL & 449.12 \\
Third and subsequent years \\
of follow-up
\end{tabular}

screenings; c) the cost of additional procedures prompted by false-positive tests. Moreover, we considered the direct cost of overdiagnosed LCs, which was based on the estimate that in the Mayo Lung Project approximately $5 \%$ of screen-detected LCs were overdiagnosed [3].

\section{Cost of LC management on the screening-invited cohort}

For each of the 245 LC cases that were diagnosed overall in the cohort [1], we computed all healthcare costs for LC management, from the time of diagnosis until the time of death. For subjects still alive at the end of study, the computation of costs was truncated at the study cut off, on December 31, 2006. Briefly, to calculate the total cost of each LC case in the cohort, costs related to LC diagnosis, staging, treatment and follow-up procedures (tables 1-3) that were individually incurred were included, stratified by stage, histology (non-small cell lung cancer, small cell lung cancer) and treatment (surgery, chemotherapy, radiation). To determine the price of the component services, we considered the phase of care and the frequency of cost-generating follow-up procedures for each case. For components with little variability of cost in the various different NHS facilities throughout the Varese province, the average cost was indicated; if the overall cost was considerably variable, the lowest value and the highest value were taken (tables 1-3).

\section{Cost of LC management in the control group}

As a control group we used a previously described group of 156 uninvited and unscreened LC patients diagnosed during the 2000 calendar year in the Varese district area, who possessed the necessary screening criteria [1]. Over the 9.5year timeframe we computed the total direct cost of healthcare procedures and interventions (tables 1-3) incurred for LC management for these 156 control group patients, who received the NHS usual standard of care in the absence of screening.

\section{Results}

\section{Cost of the screening programme}

The total direct cost of the screening programme over the 9.5-year period from July 1997 through December 2006 was estimated to range from $€ 390,934$ to $€ 394,042$ (table 4), after adding up the cost components a), b), c), as follows:

\section{a) Organization and administration}

The general expenses incurred for the initial organization of the screening programme comprised: office supplies; printed recruitment forms and brochures; mailing of screening invitation letters; advertising of screening with posters and announcements in local newspapers, radio and TV; purchase of personal computers to store CXR readings in the 7 NHS radiologic centers collaborating in the project; dedicated software for database in the project main office. Cost of programme administration included: part-time secretary; office utilities and material supplies; staff meetings. Total cost of organization and administration over the 9.5 years was $€$ 242,632 .

Table 4. - Cost of screening programme, period July 1997 - December 2006

\begin{tabular}{|c|c|}
\hline Components of cost & Cost in Euros* \\
\hline Organization and administration & 242,632 \\
\hline Total cost of CXR (5,581 screens) & 86,450 \\
\hline \multicolumn{2}{|l|}{$\begin{array}{l}\text { Additional procedures prompted } \\
\text { by screening }\end{array}$} \\
\hline $\begin{array}{l}\text { - Chest CT with contrast } \\
\text { (190 false positives) }\end{array}$ & 30,615 \\
\hline $\begin{array}{l}\text { - CT-guided fine needle biopsy } \\
\text { (1 subject) }\end{array}$ & 157 \\
\hline $\begin{array}{l}\text { - Thoracoscopic lung wedge biopsy } \\
\text { (8 subjects)* }\end{array}$ & $31,080-34,188$ \\
\hline Total* & 390,934 - 394,042 \\
\hline
\end{tabular}


b) CXR screens

Total expenditure for the 5,581 screening tests performed on the screening participants (baseline, $\mathrm{n}=1,244$; annual screens, $\mathrm{n}=4,337$ ) was $€ 86,450$.

\section{c) Additional procedures prompted by screening}

Uncertain or false-positive tests [1] prompted the following additional investigations: 190 chest computed tomography (CT) exams with contrast; one fine-needle aspirate cytology of lung; 8 thoracoscopic lung wedge resections for biopsy. The total cost of these additional procedures, none of which caused severe complications, ranged from $€ 61,852$ to $€ 64,960$.

\section{Cost of LC management in the screening-invited cohort}

The total direct cost incurred for healthcare of all LC cases diagnosed in participants and in nonparticipants of the invited cohort is shown in table 5 , with the indication of the lowest and highest value of services. The total direct cost of all 245 LC cases of the screening-invited cohort ranged from $€ 2,959,155$ to $€ 3,324,662$ (table 5). The latter cost will subsequently be compared in table 5 with the total direct cost of only 243 LCs of the control group, as 2 LCs in the invited cohort were likely to have been overdiagnosed (see below).

\section{Overdiagnosed LCS}

As 34 LCs were screen-detected in our screening programme [1], we approximated that 2 LCs $(5 \%)$ had been overdiagnosed. Accordingly during the calculation of the incremental costs of screening, to account for overdiagnosed LCs, the total direct cost of only 243 control group LCs will be subtracted from the cost of the 245 LCs from the screening-invited cohort (see calculation in table 5).

\section{Total cost of screening and LC management in the screening-invited cohort}

The cost of the screening programme added to the cost of LC management of all 245 cases in the screening-invited cohort totaled between $€$ $3,350,089$ and $€ 3,718,704$ (computation shown in table 5).

\section{Cost of LC management in the control group}

The mean direct cost of LC management for each patient of the control group was estimated to range from $€ 11,809$ to $€ 13,361$, implying a total expenditure ranging from $€ 2,869,615$ to $€$ $3,246,723$ for the 243 LCs from the control group (table 5). The total cost of LC management was slightly lower in the control group compared to the invited cohort, as in the latter there were 2 overdiagnosed cases treated and higher number of stage I surgically treated patients (table 5).

\section{Incremental costs of LC management including screening}

In the invited cohort the cumulative incremental costs of LC management, including screening, was estimated to range between $€ 471,981$ and $€$ 480,474 over the 9.5 -year study period (see calcu-

Table 5. - Lowest and highest direct cost $\left(\right.$ Euros $\left.^{*}\right)$ estimated for management of lung cancer (LC) cases during the 9.5 years of study (July 1997- December 2006)

\begin{tabular}{|c|c|c|c|c|}
\hline & \multicolumn{2}{|c|}{$\begin{array}{l}\text { LCs of screening invited cohort } \\
\qquad(n=245)\end{array}$} & \multicolumn{2}{|c|}{$\begin{array}{l}\text { LCs of control group } \\
\qquad(\mathrm{n}=243)^{\wedge}\end{array}$} \\
\hline & Lowest cost & Highest cost & Lowest cost & Highest cost \\
\hline (A): Cost of the screening programme \# & 390,934 & 394,042 & 0,00 & 0,00 \\
\hline (B): Cost of LC management & $2,959,155$ & $3,324,662$ & $2,869,615$ & $3,246,723$ \\
\hline $\begin{array}{l}{[(A)+(B)]: \text { Total cost of screening }} \\
\text { and LC management }\end{array}$ & $\begin{array}{l}3,350,089 \\
\text { (a) }\end{array}$ & $\begin{array}{l}3,718,704 \\
\text { (b) }\end{array}$ & $\begin{array}{l}2,869,615 \\
\text { (c) }\end{array}$ & $\begin{array}{l}3,246,723 \\
\text { (d) }\end{array}$ \\
\hline $\begin{array}{l}\text { Incremental cost of LC management } \\
\text { including screening }\end{array}$ & $\begin{array}{l}480,474 \\
\text { (a)-(c) }\end{array}$ & $\begin{array}{l}471,981 \\
\text { (b)-(d) }\end{array}$ & & \\
\hline $\begin{array}{l}\text { Incremental cost of LC management } \\
\text { including screening (Euros 2012) }\end{array}$ & 618,370 & 607,440 & & \\
\hline $\begin{array}{l}\text { Incremental cost per one LC death averted } \\
\text { with screening (Euros 2012) @ }\end{array}$ & 16,273 & 15,985 & & \\
\hline
\end{tabular}

LC: lung cancer.

* Costs in the table are in Euros 2002 (the central year of the study), except in the last two lines where the incremental cost incurred with screening is shown after conversion in Euros 2012 (x 1.287, based on ISTAT healthcare price index).

$\wedge$ Cost was calculated for 243 cases because 2 cases were estimated to be overdiagnosed (see text).

\# Cost of administration, CXRs and false-positives management.

@ A total of 38 LC deaths were averted during the 9.5 years of study. 
lation in table 5). After conversion based on the ISTAT healthcare price index, this corresponds to a total of between $€ 607,440$ and $€ 618,370$ as of 2012 (approx. $€ 63,941$ - $€ 65,092$ per annum). Accordingly, the incremental costs of LC management including screening ranged between $€$ 15,985 and $€ 16,273$ for each of the 38 LC deaths averted in the cohort.

In conclusion, in a general population of 60,000 adults we carried out an annual CXR screening program targeted at 5,815 high-risk smokers, and found that the overall healthcare expense for LC management including screening increased approximately by $€ 65,000$ per annum.

\section{Discussion}

It has long been recognized that $\mathrm{LC}$ has better prognosis when it is incidentally detected during routine CXR examinations. LCs when incidentally diagnosed are frequently at an early stage and are resectable, meanwhile symptom-detected cases are predominantly advanced stage cases and are rarely curable [4-6]. For this reason chest radiograph, a widely accessible and relatively inexpensive examination, is intuitively appealing as a screening tool for early stage LC diagnosis in subjects with an increased risk of death from LC such as smokers and former smokers. However, the numerous randomized trials carried out to evaluate CXR screening for LC have brought only mixed results [7-10] and present important methodological weaknesses $[2,5,7,9,11]$. All of the randomized trials have been criticized due to a self-selection bias of volunteers [7, 12], and there still remains uncertainty regarding the value of LC screening at population level in the real world [2]. Accordingly the U.S. Preventive Services Task Force stated that the evidence provided, whether it be for or against CXR screening, is insufficient [13].

Nevertheless, several authors have set out to develop LC screening programs and cost-effectiveness models with sensitivity analyses [6, 14, 15]. These analyses are quite difficult to interpret. Models of simulated cohorts have provided highly variable estimates, ranging from highly cost-effective to non cost-effective treatment methods [1417], and it remains uncertain whether a public healthcare system can afford the financial burden of population-based screening for LC.

In 1997 we started a population-based programme consisting of annual CXR screenings in the cohort of high-risk smokers who were identified within a general population of 60,000 adults from the Varese province. After the 9.5 year observation period we subsequently reported that within the screening-invited cohort, LC mortality had reduced by $18 \%$ [2]. At this point we analyzed the direct costs of our screening programme. For the entire cohort of 5,815 screening-invited smokers (21\% of whom participated) [12], the incremental cost (as of 2012) of LC management including screening was estimated to range between $€ 607,440$ and 618,370 (approximately $€ 65,000$ per annum). As 38 fewer LC deaths were recorded in the invited cohort, the additional cost of screening to avoid one LC death (when compared with no screening and after accounting for overdiagnosis) was approximately $€ 16,000$. The latter cost favourably compares with the recently estimated $\$ 240,000$ additional cost of screening with CT to avert one LC death [6], a ten-fold cost increase. Our study has limitations. First of all, cost estimates calculated in 2002 were applied to the whole period from July 1997 through December 2006. Because 2002 was the central year of our programme, it is highly unlikely that the overall cost of healthcare for all LCs in the study was markedly either under- or overestimated. Secondly, an element of cost was waived in our analysis, possibly causing screening program costs to be slightly underestimated: the screening programme main office was made available free of charge on NHS premises at the Center for Thoracic Surgery of University of Insubria, at the Varese Ospedale di Circolo. A strong feature of this study is the analytical computation based on all costs actually incurred during screening and LC management in all individual cases which occurred both within the invited cohort and the control group in the NHS setting in Varese. With emphasis of the current study being on cost, a complete cost-effectiveness assessment of the screening programme carried out on smokers remains to be done. However, the quantification of all the direct costs we incurred during our population-based CXR screening programme may be used by health policy makers to better explore the NHS budget impact and economic sustainability of LC screening.

Acknowledgements: This study was funded in part by Associazione PREDICA onlus, by the University of Insubria FAR1997-FAR2007 and by the Varese Lions Clubs Host and District $108 \mathrm{Ib}-1$.

We gratefully acknowledge the support and assistance provided by the Amare no-profit organization Como, Italy and by the Paola Giancola Foundation for Cancer Research Como, Italy.

\section{References}

1. Dominioni L, Rotolo N, Mantovani W, et al. A population-based cohort study of chest $\mathrm{x}$-ray screening in smokers: lung cancer detection findings and follow-up. BMC Cancer 2012; 12: 18.

2. Dominioni L, Poli A, Mantovani W, et al. Assessment of lung cancer mortality reduction after chest X-ray screening in smokers: a population-based cohort study in Varese, Italy. Lung Cancer 2013; 80: 50-4.

3. Yankelevitz DF, Kostis WJ, Henschke CI, et al. Overdiagnosis in chest radiographic screening for lung carcinoma: frequency. Cancer 2003; 97: 1271-5.

4. Guiss LW. A 5-year follow-up of roentgenographically detected lung cancer suspects. Cancer 1960; 13: 82-90.

5. Spiro SG. Screening for lung cancer: yet another problem. Thorax 2007; 62: 105-6.

6. Goulart BH, Bensink ME, Mummy DG, Ramsey SD. Lung cancer screening with low-dose computed tomography: costs, national expenditures, and cost-effectiveness. J Natl Compr Canc Netw 2012; 10: 267-75.

7. Humphrey LL, Teutsch S, Johnson M; U.S. Preventive Services Task Force. Lung cancer screening with sputum 
cytologic examination, chest radiography, and computed tomography: an update for the U.S. Preventive Services Task Force. Ann Intern Med 2004; 140: 740-53.

8. Church TR. Chest radiography as the comparison for spiral CT in the National Lung Screening Trial. Acad Radiol 2003; 10: 713-5.

9. Manser R, Irving LB, Stone C, Byrnes G, Abramson M, Campbell D. Screening for lung cancer. Cochrane Database Syst Rev 2004; CD001991.

10. Sox HC. Screening for lung cancer with chest radiographs. JAMA 2011; 306: 1916-8.

11. Dominioni L, Poli A, Mantovani W, Rotolo N, Imperatori A. Volunteer effect and compromised randomization in the Mayo Project of screening for lung cancer. Eur J Epidemiol 2011; 26: 79-80.

12. Dominioni L, Rotolo N, Poli A, et al. Self-selection effects in smokers attending lung cancer screening. A 9.5year population-based cohort study in Varese, Italy. $J$ Thorac Oncol 2010; 5: 428-35.
13. U.S. Preventive Services Task Force. Lung cancer screening: Recommendation statement. Ann Intern Med 2004; 140: 738-9.

14. Manser R, Dalton A, Carter R, Byrnes G, Elwood M, Campbell DA. Cost-effectiveness analysis of screening for lung cancer with low dose spiral CT (computed tomography) in the Australian setting. Lung Cancer 2005; 48: 171-85.

15. McMahon PM, Kong CY, Bouzan C, et al. Cost-effectiveness of computed tomography screening for lung cancer in the United States. J Thorac Oncol 2011; 6: 1841-8.

16. Mahadevia PJ, Fleisher LA, Frick KD, Eng J, Goodman SN, Powe NR. Lung cancer screening with helical computed tomography in older adult smokers: a decision and cost-effectiveness analysis. JAMA 2003; 289: 313-22.

17. Wisnivesky JP, Mushlin AI, Sicherman N, Henschke C. The cost-effectiveness of low-dose CT screening for lung cancer: preliminary results of baseline screening. Chest 2003; 124: 614-21.

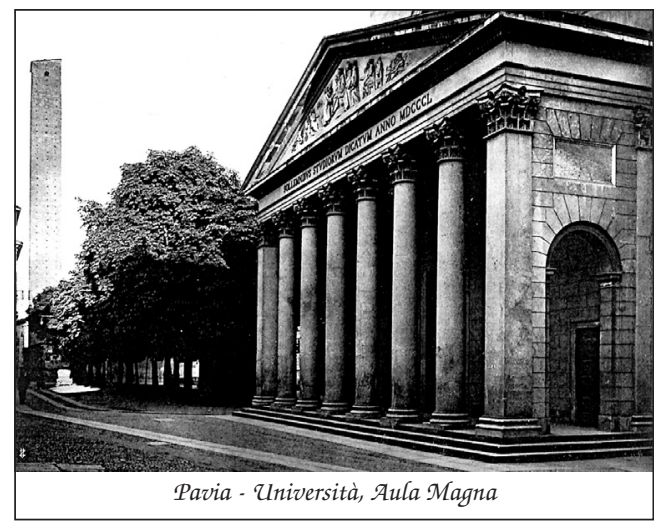

